\title{
Efficiency and Benchmarking in The Presence of Undesirable (Bad) Outputs: A DEA Approach
}

\author{
G.R. Jahanshahloo, F. Hosseinzadeh Lotfi, R. Maddahi and Y. Jafari \\ Department of Mathematics, Science and Research Branch, \\ Islamic Azad University, Tehran, Iran \\ Email:jahanshahloomath@gmail.com \\ hosseinzadeh_lotfi@yahoo.com \\ Reza.Maddahi.esf@gmail.com \\ yasserjafari2006@gmail.com
}

\begin{abstract}
Environmental assessment recently becomes a major policy issue in the world. This study introduces a data envelopment analysis (DEA) model to explore a new use of DEA for the environmental assessment in which outputs are classified into desirable (good) and undesirable (bad). Such an output separation is important in the DEA-based environmental assessment. We used a Range-Adjusted Measure (RAM) DEA model for combining the two performance measures operational and environmental performance as a unified measure. Reducing the amount of undesirable outputs is the main purpose of environmental assessment. Therefor, we try to project the inefficient organization on the efficient frontier such that the obtained projection produces fewer undesirable outputs than the evaluated organization. To reach this goal, it is not important to increase or decrease the amount of inputs.
\end{abstract}

Keywords: Data envelopment analysis, Environmental assessment, Range adjusted measure, Undesirable outputs.

\section{Introduction}

Data Envelopment Analysis (DEA) has been long serving as a methodology to evaluate the performance of various organizations or Decision Making Units (DMU). It was first proposed by Charnes et al.[1]. DEA has already been 
applied in the Environmental assessment that has recently become a major policy issue in the world. In the classical production possibility sets, DMUs consume inputs to produce outputs but in the environmental production possibility sets, DMUs consume inputs to produce outputs that may be classified into desirable (good) and undesirable (bad). The main question in this case is "How to deal with undesirable outputs?" Many papers have tried to answer this question, some of which are mentioned here. Fare et al. [3] implemented the nonparametric approach on a 1976 data set of 30 US mills which use pulp, and three other inputs in order to produce paper and four pollutants. In their research, they assumed weak disposability ${ }^{1}$ for undesirable outputs. Their results showed that the performance rankings of DMUs turned out to be very sensitive to whether or not undesirable outputs were included. (Yang and Pollitt [6]). Yaisawarng and Klein [18] constructed a DEA model to measure the effects of $\mathrm{SO}_{2}$ control on the efficiency change of US coal fired power plants in the 1980s. They assumed weak disposability for undesirable outputs, too. Fare et al. [4] introduced an environmental performance indicator by decomposing overall productivity into an environmental index and a productive efficiency index. Hongliang Yang and Michael Pollitt [6] incorporated both undesirable outputs and uncontrollable variables into DEA. They constructed a DEA model to evaluate the performance of Chinese coal-fired power plants. Jahanshahloo et al. [5] proposed a non-radial DEA model in order to improve the performance of an inefficient Decision Making Unit (DMU) in the presence of undesirable outputs, and they supposed that there exist undesirable inputs, too. Toshiyuki Sueyoshi et al. [7] proposed a new DEA approach to evaluate the operational, environmental and both-unified performance (three DEA efficiencies) of US coal-fired power plants. Also, they have developed their own method with several papers, See [7-17] for more information.

This study introduces a non-radial DEA model to explore a new use of DEA for the environmental assessment in which outputs are classified into desirable (good) and undesirable (bad). Efficiency analysis is performed not only to estimate the current level of efficiency, but also to provide information on how to remove inefficiency, that is, to obtain benchmarking information. DEA was developed in order to satisfy both objectives and the strength of its benchmarking analysis gives DEA a unique advantage over other methodologies of efficiency analysis. Since reducing the amount of undesirable outputs is the main purpose of environmental assessment, we are looking to find a projection (a benchmark) on the operational efficient frontier for the inefficient DMU that produces fewer undesirable outputs from the DMU under evaluation. To reach this goal, it is not important to increase or decrease the amount of inputs. we used a Range-Adjusted Measure DEA model that was first proposed by

\footnotetext{
${ }^{1}$ Weak disposability of outputs implies that given an input vector $\mathrm{x}$, if $y$ can be produced, then $\beta y, 0 \leq \beta \leq 1$ can also be produced.
} 
Cooper et al. [2] for combining the two performance measures operational and environmental performance as a unified measure.

The remainder of this paper has the following structure: In Section2, we describe our methodology. Section3 illustrates the proposed method using an example. Finally, conclusions are given in Section4.

\section{Methodology}

Consider $n$ decision making units $D M U_{j},(j=1,2, \ldots, n)$, each $D M U_{j}$ consuming input levels $x_{i j}>0,(i=1,2, \ldots, m)$ to produce two kinds of desirable (good) outputs $y_{r j}>0,(r=1,2, \ldots, s)$ and undesirable (bad) outputs $u_{l j}>0$, $(l=1,2, \ldots, h)$.

\subsection{Determining operational efficiency}

In this section, we propose a Range-Adjusted Measure model for determining operational efficiency of the pth organization. The formulation is as follows:

$$
\begin{aligned}
& \operatorname{Max} \sum_{r=1}^{s} R_{r}^{g} s_{r}^{g}+\sum_{i=1}^{m} R_{i}^{g} s_{i}^{x g} \\
& \sum_{j=1}^{\text {s.t }} x_{i j} \lambda_{j}+s_{i}^{x g}=x_{i p} \quad(i=1,2, \ldots, m) \\
& \sum_{j=1}^{n} y_{r j} \lambda_{j}-s_{r}^{g}=y_{r p} \quad(r=1,2, \ldots, s) \\
& \sum_{j=1}^{n} \lambda_{j}=1 \\
& s_{i}^{x g} \geq 0 \quad(i=1,2, \ldots, m) \\
& s_{r}^{g} \geq 0 \quad(r=1,2, \ldots, s)
\end{aligned}
$$

Where superscript $(\mathrm{g})$ is incorporated in order to specify desirable (good) outputs. $\lambda_{j}$ indicates the $j$ th intensity variable. $s_{i}^{x g},(i=1,2, \ldots, m)$ is the $i$ th slack variable related to ith input, and $s_{r}^{g},(r=1,2, \ldots, s)$ are all slack variables related to desirable outputs. Also, the upper and lower bounds of each desirable output, are mathematically expressed by: $\bar{y}_{r}=\max _{j}\left\{y_{r j}\right\}$, $\underline{y}_{r}=\min _{j}\left\{y_{r j}\right\}$ and the upper and lower bounds of each input, are mathematically expressed by: $\bar{x}_{i}=\max _{j}\left\{x_{i j}\right\}, \underline{x}_{i}=\min _{j}\left\{x_{i j}\right\}$ respectively. Then, $R_{r}^{g}=\frac{1}{\left[(m+s)\left(\bar{y}_{r}-\underline{y}_{r}\right)\right]}$ for all $r$ and $R_{i}^{x}=\frac{1}{\left[(m+s)\left(\bar{x}_{i}-\underline{x}_{i}\right)\right]}$ for all $i$ indicate the ranges for desirable outputs and inputs, respectively. An operational efficiency score $\left(\varphi_{1}\right)$ is measured on the optimality of Model (1) as below :

$$
\varphi_{1}=1-\left(\sum_{i=1}^{m} R_{i}^{x} s_{i}^{x g *}+\sum_{r=1}^{s} R_{r}^{g} s_{r}^{g *}\right)
$$

where $(*)$ denotes optimal values in Model (1). Here, the equation indicates the level of unified efficiency by subtracting the level of inefficiency from unity. 
In Model (1), an inefficient DMU improves its operational performance by decreasing the amount of its inputs and increasing the amount of desirable outputs as much as possible.

\subsection{Determining environmental efficiency}

In this section, we introduce a non-radial DEA model to explore a new use of DEA for the environmental assessment in which outputs are classified into desirable (good) and undesirable (bad). We evaluate the environmental efficiency of the pth DMU by using the following Range-Adjusted Measure model

$\operatorname{Max} \sum_{l=1}^{h} R_{l}^{b} s_{l}^{b}$

$\sum_{j=1}^{\mathrm{s} . \mathrm{t}} x_{i j} \lambda_{j}+s_{i}^{x g}-s_{i}^{x b}=x_{i p} \quad(i=1,2, \ldots, m)$

$\sum_{j=1}^{n} \lambda_{j}=1$

$\sum_{j=1}^{n} u_{l j} \lambda_{j}+s_{l}^{b}=u_{l p} \quad(l=1,2, \ldots, h)$

$\lambda_{j} \geq 0 \quad(j=1,2, \ldots, n)$

$s_{i}^{x g} \geq 0 \quad(i=1,2, \ldots, m)$

$s_{i}^{x b} \geq 0 \quad(i=1,2, \ldots, m)$

$s_{l}^{b} \geq 0 \quad(l=1,2, \ldots, h)$

Where superscripts ( $g$ and $b$ ) are incorporated in order to specify desirable (good) outputs and undesirable (bad) outputs, respectively. The ranges incorporated in Model (2) are specified as follows : $R_{l}^{b}=\frac{1}{h\left(\bar{u}_{l}-\underline{u}_{l}\right)}$ for all $l$. $s_{i}^{x g}, s_{i}^{x b},(i=1,2, \ldots, m)$ are all input slack variables related to desirable and undesirable outputs, respectively. $s_{l}^{b},(l=1,2, \ldots, h)$ are all slack variables related to undesirable outputs.

In Model (2), we evaluate environmental performance of DMUp, such that an arbitrary increase or a decrease in inputs can occur as long as undesirable outputs decrease. In fact Model (2) tries to reduce the undesirable outputs regardless of the amount of inputs. Since the reduction of undesirable outputs in the environmental assessment is important and we believe that between the two organizations that produce the same desirable outputs, one is more efficient that produces fewer undesirable outputs (even it consumes more inputs). Therefor, Model (2) focused on reducing them. Also, Model (2) projects the DMU under evaluation on the environmental efficiency frontier. So, an environmental inefficiency score of the DMUp is measured by Model (2) and then the environmental efficiency score $\left(\varphi_{2}\right)$ is:

$$
\varphi_{2}=1-\left(\sum_{l=1}^{h} R_{l}^{b} s_{l}^{b *}\right)
$$


where the superscript $(*)$ indicates the optimality of Model (2).

\subsection{Benchmarking in the presence of undesirable out- puts}

The main purpose of this section is to introduce an appropriate benchmark in the presence of undesirable outputs. At first, we list the properties of one appropriate benchmark.

property 1. An appropriate benchmark has to produce fewer undesirable outputs than the evaluating DMU.

property 2. An appropriate benchmark should be on the operational efficiency frontier.

The arrangement of the previous list is important. So, in the following model, we first try to decrease the amount of undesirable outputs without any attention to the amount of inputs, maybe they decrease or increase. Then, we try to increase the amount of desirable outputs and decrease the amount of inputs without any change in the amount of the current level of undesirable outputs.

$\operatorname{Max} \sum_{l=1}^{h} R_{l}^{b} s_{l}^{b}+\varepsilon\left(\sum_{i=1}^{m} R_{i}^{x} s_{i}^{x g}+\sum_{r=1}^{s} R_{r}^{g} s_{r}^{g}\right)$

$\sum_{j=1}^{\mathrm{S} . \mathrm{t}} x_{i j} \lambda_{j}+s_{i}^{x g}-s_{i}^{x b}=x_{i p} \quad(i=1,2, \ldots, m)$

$\sum_{j=1}^{n} y_{r j} \lambda_{j}-s_{r}^{g}=y_{r p} \quad(r=1,2, \ldots, s)$

$\sum_{j=1}^{n} \lambda_{j}=1$

$\sum_{j=1}^{n} u_{l j} \lambda_{j}+s_{l}^{b}=u_{l p} \quad(l=1,2, \ldots, h)$

$\lambda_{j} \geq 0 \quad(j=1,2, \ldots, n)$

$s_{i}^{x g} \geq 0 \quad(i=1,2, \ldots, m)$

$s_{i}^{x b} \geq 0 \quad(i=1,2, \ldots, m)$

$s_{l}^{b} \geq 0 \quad(l=1,2, \ldots, h)$

The ranges incorporated in Model (3) are specified as follows : $R_{l}^{b}=\frac{1}{(m+s+h)\left(\bar{u}_{l}-\underline{u}_{l}\right)}$ for all $l$ indicate the ranges for undesirable outputs. $R_{r}^{g}=\frac{1}{\left[(m+s+h)\left(\bar{y}_{r}-\underline{y}_{r}\right)\right]}$ for all $r$ and $R_{i}^{x}=\frac{1}{\left[(m+s+h)\left(\bar{x}_{i}-\underline{x}_{i}\right)\right]}$ for all $i$ indicate the ranges for desirable outputs and inputs, respectively. $s_{r}^{g},(r=1,2, \ldots, s)$ are all slack variables related to undesirable outputs. Other parameters and variables are defined exactly as before. Notice that Model (3) evaluates the environmental and operational efficiency $(\theta)$ of the DMUp at the same time, so we call it "unified efficiency score" and it is equal: 


$$
\theta=1-\left(\sum_{l=1}^{h} R_{l}^{b} s_{l}^{b *}+\sum_{i=1}^{m} R_{i}^{x} s_{i}^{x g *}+\sum_{r=1}^{s} R_{r}^{g} s_{r}^{g *}\right)
$$

Where the superscript $\left(^{*}\right)$ indicates the optimality of Model (3). Also, this model produces a projection (benchmark) for $D M U p$, that is :

$$
\left(\sum_{j=1}^{n} x_{j} \lambda_{j}^{*}, \sum_{j=1}^{n} y_{j} \lambda_{j}^{*}, \sum_{j=1}^{n} u_{j} \lambda_{j}^{*}\right) .
$$

Where the Superscript $(*)$ denotes optimal value of Model (3). Here, $x_{j}=$ $\left(x_{1 j}, x_{2 j}, \ldots, x_{m j}\right)^{T}, y_{j}=\left(y_{1 j}, y_{2 j}, \ldots, y_{s j}\right)^{T}$ and $u_{j}=\left(u_{1 j}, u_{2 j}, \ldots, u_{h j}\right)^{T}$ are column vectors of inputs, desirable outputs and undesirable outputs, respectively.

As shown in Fig 1. at first Model (3) projects DMUp on the part of environmental efficiency frontier included between point $\mathrm{A}$ and point B. For example DMUp can be projected on point $\mathrm{C}$ that consumes more input and produces less undesirable output. Then it projects point $\mathrm{C}$ on the operational efficiency frontier.

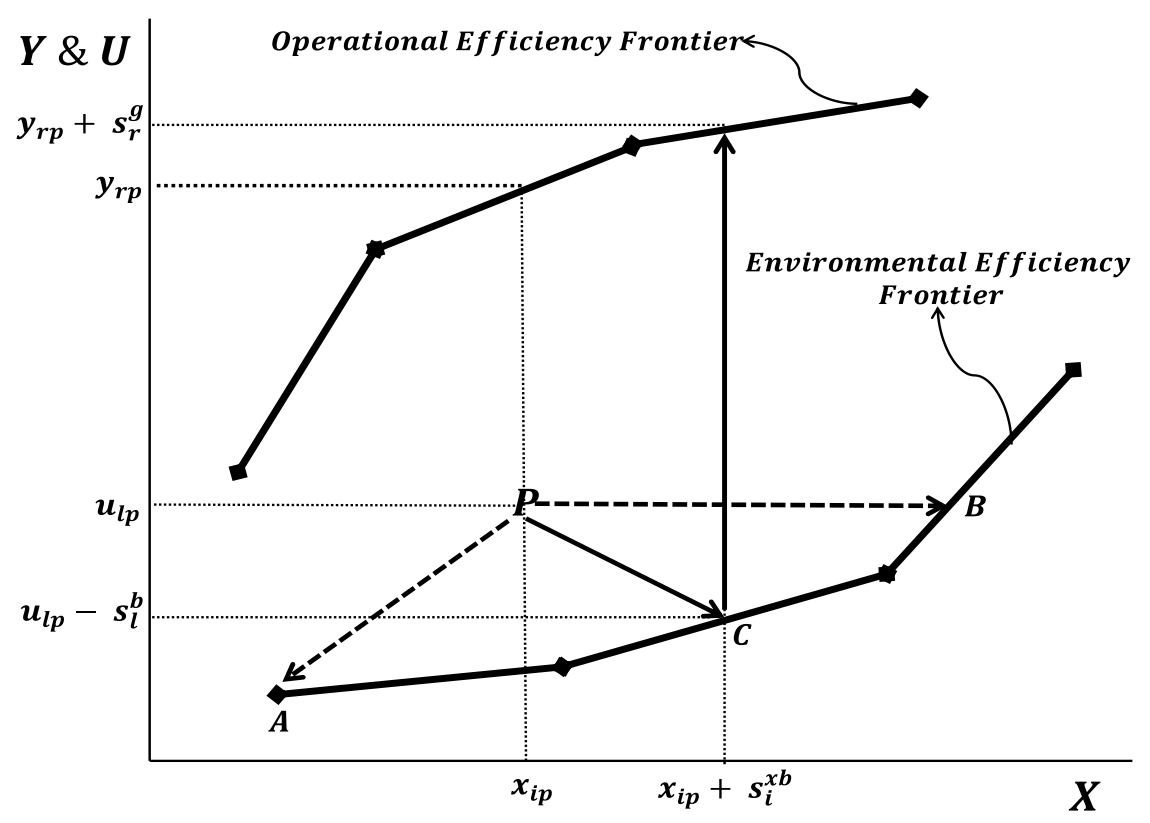

Figure 1: Environmental Efficiency Projection.

\section{$3 \quad$ Numerical example}

To illustrate our approach, numerical illustration is done regarding the example that was used by Sueyoshi and Goto [14]. They selected a small example 
Table 1: Electric power firms.

\begin{tabular}{cccccc}
\hline (Electric Power Company) DMU & $x_{1}$ & $x_{2}$ & $y_{1}$ & $y_{2}$ & $u$ \\
\hline \hline (Hokkaido) A & 15.6 & 5.7 & 318.4 & 39.4 & 167.8 \\
(Tohoku) B & 36.8 & 12.4 & 811 & 76.8 & 397.9 \\
(Tokyo) C & 129.9 & 37.9 & 2889.6 & 284.9 & 1265.0 \\
(Chubu) D & 51.1 & 16.2 & 1297.3 & 104.6 & 646.7 \\
(Hokuriku) E & 14.2 & 4.6 & 281.5 & 20.8 & 185.2 \\
(Kansai) F & 62.4 & 22.1 & 1458.7 & 134 & 549.9 \\
(Chugoku) G & 26.1 & 9.9 & 612.2 & 51.9 & 430.7 \\
(Shikoku) H & 13.5 & 6.0 & 287.0 & 28.3 & 114.6 \\
(Kyushu) I & 38.3 & 12.5 & 858.8 & 84.0 & 341.0 \\
\hline
\end{tabular}

related to Japanese electric power companies which produce more than $25 \%$ of $\mathrm{CO}_{2}$ of the whole Japanese emission. Table 1. summarizes the data set on their performance which consist of two inputs (i.e., the total amount of assets and the number of employees), two desirable outputs (i.e., the total amount of sales and the number of customers) and an undesirable output (i.e., the total amount of $\mathrm{CO}_{2}$ emission). The Japanese electric power industry consists of nine investor-owned electric power firms, all of which are vertically integrated from generation to retail supply of electricity where the input, output and undesirable variables are defined as follows:

$\left(x_{1}\right)$ : Input1 Total assets (100 billion JPY)(JPY stands for Japanese Yen).

$\left(x_{2}\right)$ : Input2 Number of employees (1000) .

$\left(y_{1}\right)$ : Desirable Output1 Total sales $(100 \mathrm{GWH})(\mathrm{GWh}$ stands for Gigawatt hours).

$\left(y_{2}\right)$ : Desirable Output2 Number Of Customers (100 Thousand).

(u): Undesirable Output $\mathrm{CO}_{2}$ emission (100Thousand ton).

In Table 2, we show three efficiency scores obtained by applying Model (1), Model (2) and Model (3) for the nine electric power companies. It is clear that for all DMUs the unified efficiency score is less than both the operational and environmental efficiency scores.

In Table 3, we show the results of Model (1).

In Table 4, we show the results of Model (2). One can see in this table that some DMUs can reduce the amount of undesirable outputs by decreasing or increasing their inputs. For example if $D M U_{B}$ decrease its Total assets with 115 billion JPY and increase its number of employees with 390 person, then it can reduce $\mathrm{CO}_{2}$ emission by 8446 Thousand tons. In a similar manner, if $D M U_{A}$ increase its Total assets and its number of employees with 284 billion JPY and 1600 person, respectively, then it can decrease $\mathrm{CO}_{2}$ emission by 808 Thousand tons. In Table 5, we show a summary of Model (3). In fact, Table 
Table 2: Three efficiency scores.

\begin{tabular}{cccc}
\hline Optimal solution & $\varphi_{1}$ & $\varphi_{2}$ & $\theta$ \\
\hline$D M U_{A}$ & 1.000 & 0.993 & 0.986 \\
$D M U_{B}$ & 0.987 & 0.927 & 0.925 \\
$D M U_{C}$ & 1.000 & 1.000 & 1.000 \\
$D M U_{D}$ & 1.000 & 0.865 & 0.851 \\
$D M U_{E}$ & 1.000 & 0.939 & 0.930 \\
$D M U_{F}$ & 0.977 & 1.000 & 1.000 \\
$D M U_{G}$ & 0.988 & 0.832 & 0.827 \\
$D M U_{H}$ & 1.000 & 1.000 & 1.000 \\
$D M U_{I}$ & 0.995 & 1.000 & 1.000 \\
\hline
\end{tabular}

Table 3: Summarize of Model (1)

\begin{tabular}{ccccc}
\hline $\mathrm{DMU}$ & $s_{1}^{x g *}$ & $s_{2}^{x g *}$ & $s_{1}^{g *}$ & $s_{2}^{g *}$ \\
\hline$D M U_{A}$ & 0.00 & 0.00 & 0.00 & 0.00 \\
$D M U_{B}$ & 0.84 & 0.68 & 68.92 & 0.00 \\
$D M U_{C}$ & 0.00 & 0.00 & 0.00 & 0.00 \\
$D M U_{D}$ & 0.00 & 0.00 & 0.00 & 0.00 \\
$D M U_{E}$ & 0.00 & 0.00 & 0.00 & 0.00 \\
$D M U_{F}$ & 0.00 & 2.95 & 9.09 & 0.00 \\
$D M U_{G}$ & 0.00 & 0.95 & 0.00 & 0.56 \\
$D M U_{H}$ & 0.00 & 0.00 & 0.00 & 0.00 \\
$D M U_{I}$ & 0.00 & 0.22 & 38.07 & 0.00 \\
\hline
\end{tabular}

Table 4: Summarize of Model (2)

\begin{tabular}{cccccc}
\hline $\mathrm{DMU}$ & $s_{1}^{x g *}$ & $s_{2}^{x g *}$ & $s_{1}^{x b *}$ & $s_{2}^{x b *}$ & $s_{1}^{b *}$ \\
\hline$D M U_{A}$ & 0.00 & 0.00 & 2.84 & 1.60 & 8.08 \\
$D M U_{B}$ & 1.15 & 0.00 & 0.00 & 0.39 & 84.46 \\
$D M U_{C}$ & 0.00 & 0.00 & 0.00 & 0.00 & 0.00 \\
$D M U_{D}$ & 0.00 & 0.00 & 4.56 & 3.68 & 200.00 \\
$D M U_{E}$ & 0.70 & 0.00 & 0.00 & 1.40 & 70.60 \\
$D M U_{F}$ & 0.00 & 0.00 & 0.00 & 0.00 & 0.00 \\
$D M U_{G}$ & 0.00 & 0.00 & 0.97 & 0.57 & 200.00 \\
$D M U_{H}$ & 0.00 & 0.00 & 0.00 & 0.00 & 0.00 \\
$D M U_{I}$ & 0.00 & 0.00 & 0.00 & 0.00 & 0.00 \\
\hline
\end{tabular}


Table 5: Summarize of Model (3)

\begin{tabular}{cccccccc}
\hline \hline $\mathrm{DMU}$ & $s_{1}^{x g *}$ & $s_{2}^{x g *}$ & $s_{1}^{x b *}$ & $s_{2}^{x b *}$ & $s_{1}^{g *}$ & $s_{2}^{g *}$ & $s_{1}^{b *}$ \\
\hline$D M U_{A}$ & 0.00 & 0.00 & 2.84 & 1.60 & 82.55 & 0.00 & 8.08 \\
$D M U_{B}$ & 1.15 & 0.00 & 0.00 & 0.39 & 0.00 & 0.00 & 84.46 \\
$D M U_{C}$ & 0.00 & 0.00 & 0.00 & 0.00 & 0.00 & 0.00 & 0.00 \\
$D M U_{D}$ & 0.00 & 0.00 & 4.56 & 3.68 & 0.00 & 14.84 & 200.00 \\
$D M U_{E}$ & 0.70 & 0.00 & 0.00 & 1.40 & 5.50 & 7.50 & 70.60 \\
$D M U_{F}$ & 0.00 & 0.00 & 0.00 & 0.00 & 0.00 & 0.00 & 0.00 \\
$D M U_{G}$ & 0.00 & 0.00 & 0.97 & 0.57 & 0.00 & 5.74 & 200.00 \\
$D M U_{H}$ & 0.00 & 0.00 & 0.00 & 0.00 & 0.00 & 0.00 & 0.00 \\
$D M U_{I}$ & 0.00 & 0.00 & 0.00 & 0.00 & 0.00 & 0.00 & 0.00 \\
\hline
\end{tabular}

5 presents the amount of changes that every DMU has to do to convert to the benchmark that produces fewer undesirable outputs as much as possible, and it is operationally efficient, too.

\section{Conclusion}

In this paper, we propose a method for evaluating a set of organizations that consume inputs to produce two kinds of desirable and undesirable outputs. Some papers recently published in this case (see Sueyoshi and Goto [7-12]) believe that for decreasing the amount of undesirable outputs, we should increase the amount of inputs. But we believe that we should focus on reducing the undesirable outputs, and to reach this goal, it is not important to increase or decrease the amount of inputs. Also, our approach determines a benchmark for the DMU under evaluation that produces fewer undesirable outputs, and it is on the operational efficient frontier, too.

\section{Acknowledgements}

The authors would like to thank the editorial team of the Journal, specially, Professor Belal Batiha for their exceptionally fast services.

\section{References}

[1] A. Charnes, W.W. Cooper, E. Rhodes, Measuring the efficiency of decision making units, Eur. J. Oper. Res. 2 (1978) 429-444.

[2] W.W. Cooper, K.S. Park, J.T. Pastor, RAM: A range adjusted measure of efficiency, Journal of Productivity Analysis. 11 (2000) 5-42. 
[3] R. Fare, S. Grosskopf, K. Lovell, C. Pasurka, Multilateral productivity comparisons when some outputs are undesirable: A nonparametric approach, The Review of Economics and Statistics. 71(1) (1989).

[4] R. Fare, S. Grosskopf, K. Lovell, C. Pasurka, Multilateral productivity comparisons when some outputs are undesirable: A nonparametric approach, The Review of Economics and Statistics. 71(1) (1989).

[5] G.R. Jahanshahloo, F. Hosseinzadeh Lotfi, N. Shoja, G. Tohidi, S. Razavyan, Undesirable inputs and outputs in DEA models, Applied Mathematics and Computation. 169 (2005) 917-925.

[6] H. Yang, M. Pollitt, Incorporating both undesirable outputs and uncontrollable variables into DEA: The performance of Chinese coal-fired power plants, Eur. J. Oper. Res. 197 (2009) 1095-1105.

[7] T. Sueyoshi, M. Goto, T. Ueno, Performance analysis of US coal-fired power plants by measuring three DEA efficiencies, Energy Policy. 38 ( 2010) 1675-1688.

[8] T. Sueyoshi, M. Goto, Should the US clean air act include $\mathrm{CO}_{2}$ emission control?: examination by data envelopment analysis, Energy Policy. 38 ( 2010) 5902-5911.

[9] T. Sueyoshi, An agent-based approach equipped with game theory: strategic collaboration among learning agents during a dynamic market change in the California electricity crisis, Energy Econ. 32 (2010) 1009-1024.

[10] T. Sueyoshi, An Agent-based approach with collaboration among agents: estimation of wholesale electricity price on PJM and artificial data generated by a mean reverting model, Energy Econ. 32 (2010) 1025-1033.

[11] T. Sueyoshi, M. Goto, Measurement of a linkage among environmental, operational and financial performance in Japanese manufacturing firms: a use of data envelopment analysis with strong complementary slackness condition, Eur. J. Oper. Res. 207 (2010) 1742-1753.

[12] T. Sueyoshi, M. Goto, DEA approach for unified efficiency measurement: assessment of Japanese fossil fuel power generation, Energy Econ. 33 ( 2011) 195-208.

[13] T. Sueyoshi, M. Goto, Methodological comparison between two unified (operational and environmental) efficiency measurements for environmental assessment, Eur. J. Oper. Res. 210 (2011) 684-693. 
[14] T. Sueyoshi, M. Goto, Measurement of returns to scale and damages to scale for DEA-based operational and environmental assessment: how to manage desirable (good) and undesirable (bad) outputs? Eur. J. Oper. Res. 211 (2011) 76-89.

[15] T. Sueyoshi, M. Goto, Returns to scale vs. damages to scale in data envelopment analysis: An impact of U.S. clean air act on coal-fired power plants, Omega: Int. J. Management Sci. (2011) (forthcoming).

[16] T. Sueyoshi, M. Goto, Data envelopment analysis for environmental assessment: Comparison between Public and Private Ownership in Petroleum Industry, Eur. J. Oper. Res. 216 (2012) 668-678 .

[17] T. Sueyoshi, M. Goto, Returns to scale and damages to scale under natural and managerial disposability: Strategy, efficiency and competitiveness of petroleum firms, Energy Econ. (2012) (forthcoming).

[18] S. Yaisawarng, J. Klein, The effects of sulfur dioxide controls on productivity change in the US electric power industry. The Review of Economics and Statistics. 76(3) (1994) 447-460. 